\title{
BMJ Open The increased risks of death and extra lengths of hospital and ICU stay from hospital-acquired bloodstream infections: a case-control study
}

\author{
Adrian G Barnett, ${ }^{1}$ Katie Page, ${ }^{1}$ Megan Campbell, ${ }^{1}$ Elizabeth Martin, ${ }^{1}$ \\ Rebecca Rashleigh-Rolls, ${ }^{1,2}$ Kate Halton, ${ }^{1}$ David L Paterson, ${ }^{3,4}$ Lisa Hall, ${ }^{1,4}$ \\ Nerina Jimmieson, ${ }^{5}$ Katherine White, ${ }^{1}$ Nicholas Graves ${ }^{1,4}$
}

To cite: Barnett AG, Page $\mathrm{K}$, Campbell M, et al. The increased risks of death and extra lengths of hospital and ICU stay from hospitalacquired bloodstream infections: a case-control study. BMJ Open 2013;3: e003587. doi:10.1136 bmjopen-2013-003587

- Prepublication history for this paper is available online. To view these files please visit the journal online (http://dx.doi.org/10.1136/ bmjopen-2013-003587).

Received 12 July 2013 Revised 22 September 2013 Accepted 3 October 2013

\section{CrossMark}

For numbered affiliations see end of article.

Correspondence to Dr Adrian G Barnett; a.barnett@qut.edu.au

\section{ABSTRACT}

Objectives: Hospital-acquired bloodstream infections are known to increase the risk of death and prolong hospital stay, but precise estimates of these two important outcomes from well-designed studies are rare, particularly for non-intensive care unit (ICU) patients. We aimed to calculate accurate estimates, which are vital for estimating the economic costs of hospital-acquired bloodstream infections.

Design: Case-control study.

Setting: 9 Australian public hospitals.

Participants: All the patients were admitted between 2005 and 2010.

Primary and secondary outcome measures: Risk of death and extra length of hospital stay associated with nosocomial infection.

Results: The greatest increase in the risk of death was for a bloodstream infection with methicillin-resistant Staphylococcus aureus ( $\mathrm{HR}=4.6,95 \% \mathrm{Cl} 2.7$ to 7.6 ). This infection also had the longest extra length of stay to discharge in a standard bed (12.8 days, $95 \% \mathrm{Cl} 6.2$ to 26.1 days). All the eight bloodstream infections increased the length of stay in the ICU, with longer stays for the patients who eventually died (mean increase 0.7-6.0 days) compared with those who were discharged (mean increase: $0.4-3.1$ days). The three most common organisms associated with Gramnegative infection were Escherichia coli, Pseudomonas aeruginosa and Klebsiella pneumonia.

Conclusions: Bloodstream infections are associated with an increased risk of death and longer hospital stay. Avoiding infections could save lives and free up valuable bed days.

\section{INTRODUCTION}

Hospital-acquired infections increase a patient's risk of death and prolong their hospital stay. ${ }^{1}$ Accurate estimates of the increased risk of death and extra length of stay are rare because of the complex statistical analysis needed to avoid the potentially

\section{Strengths and limitations of this study}

- We had an extremely large sample size but with little detailed individual information. We could not therefore match or control for detailed individual characteristics, which may mean that there is some residual confounding in our estimates.

- Our estimates will be useful for economic studies on the costs and health benefits of interventions that reduce hospital-acquired infections.

serious biases of ignoring the timing of infection. $^{2}{ }^{3}$ There are few accurate estimates of the extra length of stay and increased risk of death due to bloodstream infections (BSI), ${ }^{4}$ with most good estimates only for patients in intensive care unit (ICU). This is an important gap in our understanding of the complete burden of hospital-acquired BSIs, particularly as death and length of stay are vital for estimating the economic costs of hospital-acquired infections. ${ }^{5-7}$ Also, financial penalties are applied in some hospitals for any hospital-acquired BSI (not just central line associated BSI).

In this paper, we used an analysis that accounts for the timing of infection and hence gives accurate estimates of the risk of death and extra length of stay. We examined eight types of hospital-acquired BSIs using data from nine Australian hospitals over 6 years. We estimated the extra length of stay due to infections for standard and ICU beds.

\section{METHODS}

Data

We examined the nine largest public hospitals in Queensland, Australia (see table 1 for some descriptive statistics). We requested all 
Table 1 Basic characteristics of the nine Queensland hospitals combined, patients with admission or discharge dates between 1 January 2005 and 31 December 2010

\begin{tabular}{lrlrl}
\hline Admissions & Numbers & Patient age, median (IQR) & LoS in days, median (IQR) & In-hospital deaths (\%) \\
\hline All & 2725515 & $53(32,69)$ & $1(1,4)$ & 1.1 \\
Those with an infection & 19206 & $61(44,74)$ & $15(6,31)$ & 7.1 \\
Those without an infection & 2706309 & $53(32,69)$ & $1(1,4)$ & 1.0 \\
\hline Results for all admissions and admissions by infection status. & & \\
LoS, length of stay.
\end{tabular}

patient admissions with an admission or discharge date between 1 January 2005 and 31 December 2010 from the Health Statistics Centre of Queensland Health. The infection data came from the Centre for Healthcare Related Infection Surveillance and Prevention (CHRISP), Queensland Health. The admission and infection data were linked by the Queensland Health staff using a unique patient unit record number and infection date.

The data used included the dates of admission, discharge and infection (if any), and the dates (if any) of admissions and discharges from ICU. Data were also requested on the admitting hospital, patient's age, principal diagnosis code (international classification of diseases (ICD)-10) and outcome in three categories: discharged alive, died or censored. Censored meant the outcome of the patient was unknown, which occurred when: (1) the patient was transferred to another hospital, (2) the patient was discharged to some other facility, such as an aged care facility or medi-hotel. We accounted for this censoring in our analyses using statistical censoring.

CHRISP coordinates a state wide healthcare associated infection surveillance programme, which aggregates and assures the data quality. The surveillance definitions and processes have been refined and validated over 10 years, ${ }^{8}$ and are consistent with national and international definitions. Hospitals monitor infections hospital-wide as detailed in the surveillance manual. ${ }^{9}$ The data undergo a central quality assurance check every 6 months, and the observed numbers of infections are regularly compared with the expected numbers. Hospitals with numbers that are lower than the statewide control limit are asked about their surveillance processes.

Bloodstream infections were classified a priori into four non-mutually exclusive groups, those due to: (1) Staphylococcus aureus, (2) coagulase negative staphylococci, (3) Gram-positive organisms and (4) Gram-negative organisms. After examining the results from these four groups, we added four further subgroups. For example, $S$ aureus infections were split into methicillin-resistant $S$ aureus (MRSA) and methicillin-sensitive $S$ aureus (MSSA), and Gram-negative organisms were split into Escherichia coli and Pseudomonas aeruginosa, to examine a lower and higher virulence organism, respectively. The infection groups are not mutually exclusive. For example, BSIs due to $S$ aureus were also classified in the Gram-positive organism group.
Community associated infections were excluded. The portal of entry of BSI (eg, urinary tract infection, pneumonia, intra-abdominal infection, central line) was not available.

\section{Statistical methods}

The basis of our statistical model is shown in figure 1 . A patient's admission over time is modelled using the four states, with all patients eventually dying or being discharged, and some patients being infected. Using this multistate model, we can examine our two key questions

1. By how much did a hospital-acquired BSI increase the risk of death?

2. By how much did a hospital-acquired BSI increase the length of stay?

\section{Incidence density sampling}

We created a smaller group of infected and non-infected patients from the complete data using incidence density sampling. ${ }^{10}$ The incidence density sampling approach is illustrated in figure 2. Patient $\mathrm{E}$ is the infected case, whose infection occurred 4 days after their admission. Patient D is not a potential control, as he was discharged alive before day four. The other three patients $(\mathrm{A}-\mathrm{C}$ )

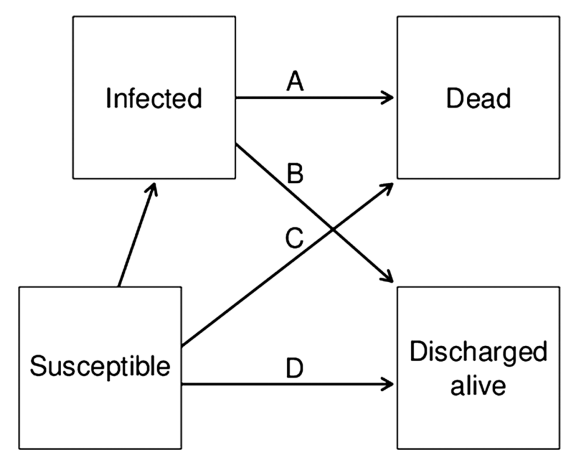

Figure 1 Four-state model to estimate the extra risk of death and extra length of stay due to a hospital-acquired bloodstream infection. The arrows represent hazards in a survival model. The extra risk of death was estimated using the HR of the hazard of death for infected patients (arrow A) and the hazard for susceptible patients (arrow $\mathrm{C}$ ). The extra length of stay for those discharged alive was calculated by comparing the time taken to discharge for infected patients (arrow B) with the time taken to discharge for susceptible patients (arrow D). 
are all eligible controls as they were infection free at the time of the case's infection. This includes patient $\mathrm{C}$, who acquired an infection on a later day.

The days in hospital after the infection (for both cases and controls) were used to estimate the extra length of stay (solid lines in figure 2). We examined the extra number of days in standard and ICU beds (thin and thick lines in figure 2, respectively). For patients with multiple infections, we only considered their first infection. This was performed to simplify the analysis (as multiple infections would require another state in figure 1), and because there were relatively few admissions with multiple infections.

Matching infected patients to control patients when estimating the extra length of stay due to infection usually gives poor estimates because of the timedependent bias. ${ }^{5}$ This bias occurs because the time before infection is used when estimating the extra length of stay (dashed horizontal lines in figure 2). However, unlike traditional matching studies, we used incidence density sampling, which also matches the timing of infection because potential controls must have been infection-free at the time of the case's infection. ${ }^{10}$

To make comparable groups of patients in terms of morbidity, we matched infected cases to controls who: had the same first letter in the principal diagnosis code (using ICD-10 coding), were of a similar age (within 10 years), were at the same hospital, and were infectionfree at the time of the case's infection. We randomly selected four controls for each of the infected patients.

\section{Statistical power}

The study had a $90 \%$ power to detect an increased HR of $1.40(40 \%)$ for infected versus uninfected patients using the smallest number of infections of 189 for MRSA, and an increased HRs of 1.18 (18\%) for the

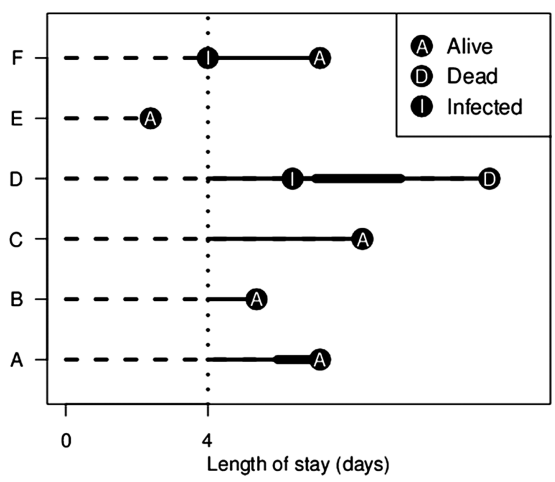

Figure 2 Illustration of incidence density sampling for an infected case (patient E) and matched controls (patients $A$ to C). The vertical dotted line shows the timing of infection. The dashed lines show the periods of hospital stay before infection. These times are discarded, as only times after infection are used to estimate the extra length of stay. The thicker solid lines show the time spent in the ICU. Adapted from Wolkewitz et al. ${ }^{10}$ second smallest number of infections of 744. These calculations assumed a two-sided 5\% significance level.

We only examined the risk of in-hospital death, as we had no information on patients after discharge.

\section{Extra length of stay}

We estimated the extra length of stay due to infection using the following steps. We calculated the number of days from infection to discharge for cases, and the number of days from the case's infection to discharge for its four matched controls. We then subtracted the case's length of stay from the average length of stay for its matched controls, with separate estimates for stays in standard and ICU beds. We then averaged these individual extra lengths of stay over all cases. These averages were stratified to create separate estimates for patients discharged alive and dead.

There are no parametric equations for calculating CIs for the extra length of stay; hence, we used a bootstrap method to generate a $95 \%$ CI. ${ }^{11}$ We randomly selected sets of cases and matched controls with replacement, creating a random sample with the same sample size as the original data. We repeated this random selection 1000 times.

All analyses were conducted in R V.2.15.0 using the 'survival' library.

\section{RESULTS}

\section{Hazard ratios}

The HRs for the eight BSIs are in table 2. All the eight infections increased the risk of death, with the largest risk for MRSA $(\mathrm{HR}=4.6)$ and the smallest for Gram-negative BSI $(\mathrm{HR}=2.1)$. The increases were statistically significant for all the eight infections, as the lower limits of the $95 \%$ CIs were all above 1 . The greatest number of infections was 2141 for Gram-positive BSI, and the smallest number was 189 for MRSA.

\section{Extra length of stay}

The extra lengths of stay for the eight BSIs are in table 3. For the patients who died, there was no extra length of stay in a standard bed (as all the 95\% CIs include 0). For the patients who were discharged alive, infection was associated with an extra length of stay in a standard bed for every type of BSI except the Gram-negative BSIs. The longest extra length of stay to discharge in a standard bed was 12.8 days for MRSA (95\% CI 6.2 to 26.1 days). The 95\% CIs are noticeably wider for infections with smaller numbers.

Most of the BSI types were associated with an extra length of stay in the ICU for the patients who lived as well as those who died (table 3). The extra lengths of stay were generally longer for those patients who died. The longest extra length of stay to death in an ICU bed was 6.0 days for a BSI with coagulase-negative staphylococci (CNS) (95\% CI 3.3 to 10.0 days). 


\begin{tabular}{|c|c|c|c|c|c|}
\hline $\begin{array}{l}\text { Bloodstream } \\
\text { infection }\end{array}$ & $\begin{array}{l}\text { Number of } \\
\text { infections }\end{array}$ & $\begin{array}{l}\text { Deaths in infections, } \\
\mathrm{n}(\%)\end{array}$ & Controls & $\begin{array}{l}\text { Deaths in controls, } \\
\mathrm{n}(\%)\end{array}$ & HR $(95 \% \mathrm{Cl})$ \\
\hline $\begin{array}{l}\text { BSI and Gram } \\
\text { positive }\end{array}$ & 2141 & $338(15.8)$ & 8512 & $526(6.2)$ & $3.0(2.6$ to 3.5$)$ \\
\hline \multicolumn{6}{|l|}{ BSI with SAB } \\
\hline All & 744 & $124(16.7)$ & 2950 & $175(5.9)$ & 3.5 (2.7 to 4.6$)$ \\
\hline MRSA & 189 & $38(20.1)$ & 740 & $45(6.1)$ & $4.6(2.7$ to 7.6$)$ \\
\hline MSSA & 555 & $86(15.5)$ & 2218 & $121(5.5)$ & 3.4 (2.5 to 4.7$)$ \\
\hline BSI with CNS & 918 & $139(15.1)$ & 3640 & $219(6.0)$ & 2.9 (2.3 to 3.7$)$ \\
\hline \multicolumn{6}{|c|}{ BSI and Gram negative } \\
\hline All & 2044 & $285(13.9)$ & 8089 & $609(7.5)$ & $2.1(1.8$ to 2.4$)$ \\
\hline Escherichia coli & 465 & 57 (12.3) & 1838 & $130(7.1)$ & 2.0 (1.4 to 2.8$)$ \\
\hline Pseudomonas & 449 & $74(16.5)$ & 1771 & $163(9.2)$ & $2.2(1.6$ to 3.0$)$ \\
\hline
\end{tabular}

Based on nine hospitals with admissions between 1 January 2005 and 31 December 2010.

BSI, bloodstream infection; CNS, coagulase-negative staphylococci; MRSA, methicillin-resistant Staphylococcus aureus; SAB, S aureus bacteraemia.

\section{CONCLUSION}

This is one of the largest studies to estimate the increased length of stay and risk of death due to hospital-acquired infection. ${ }^{4}$ All the eight BSI types studied increased the risk of death and most led to extra days in the ICU. Five of the BSIs also prolonged stay in a standard hospital bed by an average of between 9.8 and 12.8 days. The eight hospital-acquired infections studied therefore significantly increased mortality and morbidity.

Gram-negative infections had generally shorter extra lengths of stay and lower risks of death compared with the other infection types. The three most common organisms associated with Gram-negative infection were E coli, Pseudomonas aeruginosa and Klebsiella pneumoniae. There were no clear differences between patients with a Gram-positive and Gram-negative infection in terms of their age or primary diagnosis (data not shown). BSI with CNS had a higher death risk $(\mathrm{HR}=2.9)$ than Gram-negative BSIs $(\mathrm{HR}=2.1)$, which could reflect the higher risk of an organ failure. ${ }^{12}$

The average extra lengths of stay after infection were shorter for ICU bed days compared with ward bed days for all infections, which is expected as the average extra length of stay is proportional to the average total length of stay. ${ }^{2}$

MRSA was associated with the largest increased risk of death $(\mathrm{HR}=4.6)$ and the largest increase in length of stay for a standard bed (12.8 days for those discharged alive). BSI with CNS had the largest increased length of stay in an ICU bed of 6 days for patients who died and 1.4 days for patients who were discharged alive. These estimates of HR and length of stay are similar to those from related studies that account for the timedependent bias. A study in European hospitals found HRs of 3.5 due to MRSA BSI and 3.1 for MSSA BSI, with an extra length of stay of 9.2 days for MRSA BSI and 8.6 days for MSSA BSI. ${ }^{13}$ Results from ICUs in 10 European countries gave estimated HRs for BSIs ranging from 2.1 to 4.4 depending on the organism, and extra lengths of stay in the ICU ranging from 0.1 to 3.7 days. ${ }^{1}$ ICUs in France had an estimated OR for death of 3.2 due to a BSI infection, with a lower OR of 2.7 for those who received appropriate treatment. ${ }^{14}$

\section{Study limitations}

We used a large routinely collected data set of all hospital admissions. Larger data sets give more statistical power, but are often not as detailed or error-free as prospectively collected data. The hospital admission data used here are subject to data checking at the time of entry, and we subjected the data to further logical checks and found no errors.

We matched controls to cases using the first letter of the ICD-10 code so that the controls and cases were broadly similar in terms of morbidity. It is possible that even after the matching, the infected cases were sicker than the controls (prior to the infection) and that this somewhat explains the cases' extra length of stay and increased risk of death. However, adjusting for the timing of infection (which we did) is far more important than adjusting for baseline morbidity when estimating the extra length of stay due to infection. ${ }^{15}$

Despite using hospital-wide surveillance, some infections may have been missed. Surveillance relies on clinical testing, so an infected but untested patient would be missed. However, collection of blood cultures is standard for patients with a fever during hospitalisation.

Our results should be generalisable to other settings, but it is possible that differences will occur depending on how infections are managed. For example, some hospitals use hospital in the home schemes, where infected patients can be cared for at home rather than in the hospital. ${ }^{16}$ Caring for infected patients in their own home would reduce the extra length of hospital stay due to infection. Unfortunately, we did not have data on the use of hospital in the home and therefore could not estimate the entire patient journey. If we had these data, 
Table 3 Extra length of stay (in days) in a standard bed and ICU bed due to a hospital-acquired bloodstream infection

\begin{tabular}{|c|c|c|c|c|}
\hline \multirow{2}{*}{$\frac{\text { Bloodstream }}{\text { Infection }}$} & \multicolumn{2}{|l|}{ Standard bed } & \multicolumn{2}{|l|}{ ICU bed } \\
\hline & Died & Discharged & Died & Discharged \\
\hline BSI and Gram positive & $1.0(-3.9$ to 5.6$)$ & 9.8 (7.7 to 12.6$)$ & 4.0 (2.6 to 5.7$)$ & $0.9(0.4$ to 1.8$)$ \\
\hline \multicolumn{5}{|l|}{ BSI with SAB } \\
\hline All & $-1.5(-6.8$ to 6.1$)$ & $12.1(6.7$ to 15.3$)$ & $1.4(0.5$ to 3.0$)$ & $0.9(0.1$ to 2.9$)$ \\
\hline MRSA & $-1.6(-12.6$ to 12.6$)$ & $12.8(6.2$ to 26.1$)$ & 3.1 (0.5 to 7.2$)$ & 3.1 (0.4 to 13.2$)$ \\
\hline MSSA & $2.7(-2.6$ to 9.7$)$ & 11.0 (6.4 to 14.9$)$ & $0.7(-0.3$ to 2.0$)$ & 0.4 (0.0 to 0.8$)$ \\
\hline BSI with CNS & $3.5(-4.0$ to 13.4$)$ & 9.8 (3.6 to 14.6$)$ & 6.0 (3.3 to 10.0$)$ & 1.4 (0.6 to 2.5$)$ \\
\hline \multicolumn{5}{|l|}{ BSI and Gram negative } \\
\hline All & $-3.9(-8.7$ to -0.4$)$ & $2.7(-4.1$ to 6.1$)$ & $3.0(1.4$ to 4.5$)$ & 0.6 (0.3 to 1.0$)$ \\
\hline Escherichia coli & $-3.3(-9.3$ to 7.9$)$ & $1.1(-13.2$ to 5.7$)$ & $2.5(0.4$ to 4.7$)$ & $0.5(-0.1$ to 0.9$)$ \\
\hline Pseudomonas & $-5.4(-11.6$ to 9.2$)$ & $5.6(-6.4$ to 14.3$)$ & $3.2(0.8$ to 7.1$)$ & 0.5 (0.3 to 1.2$)$ \\
\hline
\end{tabular}

they could have been added as another state to the multistate model in figure 1.

We had no data on why the extra length of stay occurred. For example, the extra lengths of stay may be directly due to the increased morbidity of infection, or they could be due to a change in patient management, such as the use of defined durations of intravenous antibiotics (such as for $S$ aureus BSI). It is also possible that the total extra length of stay after a BSI is not solely due to the infection. For example, a patient's stay is initially extended because of a BSI; then during this extra stay, an unrelated adverse event happens, for example, an adverse drug reaction that keeps them in hospital for longer. ${ }^{17}$ To further investigate extra length of stay due to infection, we recommend a detailed individual study that follows patients from the time of their infection to discharge and details the decisions made and resources used. ${ }^{18}$ In some hospitals, this is already collected using a postinfection review.

\section{Study strengths}

This is one of the first studies to accurately estimate the extra length of stay due to BSI in a standard hospital bed, as most previous good estimates only examined ICU beds. This is important because days in the hospital are costly, so extra length of stay is key to determining the economic costs of infection, ${ }^{19}$ as well as being an important measure of morbidity. ICU beds have a far greater economic cost than standard beds, so it is vital to get separate estimates for ward and ICU beds. ${ }^{20}$

Our results can be used to inform parameters for studies of the cost-effectiveness of interventions that reduce risks of hospital-acquired infection. This is the most useful application of estimates, as only describing the size of the cost does not help decision-makers, although it might get the attention of politicians and the media in the short term. Also, erroneous estimates of these parameters might have misled decision-making in the past. ${ }^{5}$ The application of a multistate modelling approach (figure 1), which appropriately classifies patient risks over time, should become the gold standard method for these studies. ${ }^{3}$

A key parameter in cost-effectiveness models is the extra number of deaths, as the years of life lost have a potentially large economic cost. We found that all the eight types of BSIs increased the risk of death. Avoiding infections is therefore likely to save lives and free up valuable bed days.

\section{Author affiliations}

${ }^{1}$ Institute of Health and Biomedical Innovation, Queensland University of Technology, Queensland, Australia

${ }^{2}$ Royal Brisbane and Women's Hospital, Queensland, Australia

${ }^{3}$ The University of Queensland Centre for Clinical Research, Queensland, Australia

${ }^{4}$ Centre for Healthcare Related Infection Surveillance and Prevention, Queensland Health, Queensland, Australia

${ }^{5}$ School of Psychology, The University of Queensland, St Lucia, Queensland, Australia

Acknowledgements Thanks to all the hospital Infection Control Practitioners for undertaking the HAI surveillance used for this analysis. Thanks also to the staff at Queensland Health in the Health Statistics Centre and Centre for Healthcare Related Infection Surveillance and Prevention for providing and merging the hospital and infection data. The computational resources and services used in this work were provided by the High Performance Computer and Research Support Unit, Queensland University of Technology, Brisbane, Australia.

Contributors The study was motivated by an ongoing economic evaluation involving all the authors. AGB ran the statistical analysis and wrote the first draft and is the paper's guarantor. KP, MC, LH, DLP and NG gave critical input into the study design. All the authors read the first draft and provided edits.

Funding This work was supported by a National Health and Medical Research Council partnership grant (number 553081) with financial and in-kind support from: Australian Commission on Safety and Quality in Health Care, Hand Hygiene Australia and jurisdictional health departments. The Centre for Healthcare Related Infection Surveillance and Prevention, Communicable Diseases Branch, Queensland Health supports the salaries of NG and DLP. KP's salary comes from the National Health and Medical Research Council partnership grant. 
Competing interests None.

Ethics approval The study was approved by the ethics committees of Queensland Health and Queensland University of Technology. The Research Ethics Governance Unit for Queensland Health approved the data collection and linkage process, number: HREC/10/QPAH/180.

Provenance and peer review Not commissioned; externally peer reviewed.

Data sharing statement All the data used in this study are available from Queensland Health and the Centre for Healthcare Related Infection Surveillance and Prevention subject to ethical approval. Please contact AGB (a.barnett@qut.edu.au) if interested in accessing the data.

Open Access This is an Open Access article distributed in accordance with the Creative Commons Attribution Non Commercial (CC BY-NC 3.0) license, which permits others to distribute, remix, adapt, build upon this work noncommercially, and license their derivative works on different terms, provided the original work is properly cited and the use is non-commercial. See: http:// creativecommons.org/licenses/by-nc/3.0/

\section{REFERENCES}

1. Lambert M-L, Suetens C, Savey A, et al. Clinical outcomes of health-care-associated infections and antimicrobial resistance in patients admitted to European intensive-care units: a cohort study Lancet Infect Dis 2011;11:30-8.

2. Barnett AG, Beyersmann J, Allignol A, et al. The time-dependent bias and its effect on extra length of stay due to nosocomial infection. Value Health 2011;14:381-6.

3. Beyersmann J, Gastmeier P, Grundmann $\mathrm{H}$, et al. Use of multistate models to assess prolongation of intensive care unit stay due to nosocomial infection. Infect Control Hosp Epidemiol 2006;27:493-9.

4. Crnich $\mathrm{C}$. Estimating excess length of stay due to central line-associated bloodstream infection: separating the wheat from the chaff. Infect Control Hosp Epidemiol 2010;31:1115-17.

5. Graves N, Harbarth S, Beyersmann J, et al. Estimating the cost of health care-associated infections: mind your p's and q's. Clin Infect Dis 2010;50:1017-21.

6. Halton KA, Cook D, Paterson DL, et al. Cost-effectiveness of a central venous catheter care bundle. PLOS ONE 2010;5:e12815.
7. Graves N, Halton K, Doidge S, et al. Who bears the cost of healthcare-acquired surgical site infection? J Hosp Infect 2008;69:274-82.

8. Morton AP, Clements AC, Doidge SR, et al. Surveillance of healthcare-acquired infections in Queensland, Australia: data and lessons from the first 5 years. Infect Control Hosp Epidemiol 2008;29:695-701.

9. Centre for Healthcare Related Infection Surveillance and Prevention. Surveillance manual. Queensland Health, 2009.

10. Wolkewitz M, Beyersmann J, Gastmeier P, et al. Efficient risk set sampling when a time-dependent exposure is present. Methods Inf Med 2009;48:438-43.

11. Davison AC, Hinkley DV, Bootstrap methods and their application. Cambridge University Press, 1997.

12. Savithri MB, lyer V, Jones $M$, et al. Epidemiology and significance of coagulase-negative staphylococci isolated in blood cultures from critically ill adult patients. Crit Care Resusc 2011;13: 103-7.

13. de Kraker MEA, Wolkewitz M, Davey PG, et al. Clinical impact of antimicrobial resistance in European hospitals: excess mortality and length of hospital stay related to methicillin-resistant Staphylococcus aureus bloodstream infections. Antimicrob Agents Chemother 2011;55:1598-605.

14. Garrouste-Orgeas M, Timsit JF, Tafflet M, et al. Excess risk of death from intensive care unit-acquired nosocomial bloodstream infections: a reappraisal. Clin Infect Dis 2006;42:1118-26.

15. Beyersmann J, Kneib T, Schumacher M, et al. Nosocomial infection, length of stay, and time-dependent bias. Infect Control Hosp Epidemiol 2009;30:273-6.

16. Howden BP, Grayson ML. Hospital-in-the-home treatment of infectious diseases. Med J Aust 2002;176:440-5.

17. Hauck K, Zhao X. How dangerous is a day in hospital? A model of adverse events and length of stay for medical in patients. Med Care 2011;49:1068-75.

18. Collignon PJ, Wilkinson IJ, Gilbert GL, et al. Health care-associated Staphylococcus aureus bloodstream infections: a clinical quality indicator for all hospitals. Med J Aust 2006;184:404-6.

19. Grayson ML, Russo PL, Cruickshank M, et al. Outcomes from the first 2 years of the Australian National Hand Hygiene Initiative. Med $J$ Aust 2011;195:615-19.

20. Rechner I, Lipman J. The costs of caring for patients in a tertiary referral Australian intensive care unit. Anaesth Intensive Care 2005;33:477-82. 